05

\title{
Линейный дихроизм и двулучепреломление зондирующего излучения в спектроскопии накачка-зондирование в многоатомных молекулах
}

\author{
(C) Б.В. Семак, О.С. Васютинский
}

ФТИ им. А.Ф. Иофрфе, 194021 Санкт-Петербург, Россия

e-mail: osv@pms.ioffe.ru

Поступила в редакцию 18.12.2020 г.

В окончательной редакции 17.02.2021 г.

Принята к публикации 23.03.2021 г.

Теоретически исследованы эффекты дихроизма и двулучепреломления в возбужденных состояниях многоатомных молекул при возбуждении двумя последовательными фемтосекундными импульсами в зависимости от времени задержки между ними. Получены общие выражения для изменения интенсивности и состояния поляризации зондирующего импульса после прохождения раствора произвольных многоатомных молекул для любых исходных поляризаций каждого из лазерных импульсов. Полученные выражения для изменения поляризации зондирующего импульса записаны в представлении сферических тензорных операторов и учитывают когерентность колебательных состояний в возбужденных состояниях молекул, а также распад этих состояний за счет колебательной релаксации, вращательной диффузии и радиационных переходов. Полученные выражения содержат вклады от линейного дихроизма и двулучепреломления в возбужденных состояниях молекул. Показано, что при определенных условиях оба эффекта могут наблюдаться одновременно. Рассмотрена геометрия почти коллинеарного распространения накачивающего и зондирующего импульсов через молекулярный раствор и показано, что вклады от линейного дихроизма и двулучепреломления в сигнал могут быть полностью разделены в эксперименте за счет надлежащего выбора анализатора поляризации зондирующего импульса, установленного перед фотодетектором. Полученные выражения применены для описания сигналов, получаемых при использовании поляризационно-модуляционной экспериментальной методики, разработанной и примененной в недавней работе авторов (Gorbunova et al, Phys. Chem. Chem. Phys. 2020, Vol. 22, 18155-18168). При этом показано, что модулированные сигналы дихроизма и двулучепреломления в основном проявляются как квадратурные по отношению к опорному сигналу модуляции на двойной частоте.

Ключевые слова: дихроизм, двулучепреломление, поляризация, молекулы, лазерный импульс.

DOI: $10.21883 /$ OS.2021.07.51083.1734-21

\section{1. Введение}

В последнее время лазерная фемтосекундная спектроскопия типа накачка-зондирование (pump-probe) находит широкое применение для исследования сверхбыстрых фотопроцессов в физике, химии и биологии, поскольку она дает возможность детально и в реальном масштабе времени исследовать элементарные физические и химические процессы, происходящие в газовой и конденсированной фазах [1-3]. Лазерная фемтосекундная спектроскопия биологических молекул позволяет достичь недоступного ранее уровня понимания динамики внутри- и межмолекулярных взаимодействий, включающих релаксацию в основном и возбужденных состояниях, перенос электронов и протонов, безызлучательную рекомбинацию и другие процессы [4-6].

Быстрые релаксационные процессы, происходящие в возбужденных состояниях многоатомных молекул в растворах и живых клетках, очень часто являются анизотропными, обычно они исследуются методами многофотонной поляризованной флуоресценции [7-12] или методами поляризационной спектроскопии рumpprobe [13-21]. В обоих случаях линейно поляризованный лазерный импульс фемтосекундной длительности переводит исследуемые молекулы в возбужденное электронное состояние, причем образующийся ансамбль возбужденных молекул, как правило, характеризуется выстраиванием по направлениям молекулярных осей. Это выстраивание уменьшается со временем вследствие колебательной релаксации [21] и вращательной диффузии [7,22], а соответствующие времена затухания несут важную информацию о внутри- и межмолекулярных взаимодействиях. В рамках метода поляризованной флуоресценции, зависимость анизотропии в возбужденных состояниях молекул от времени определяется с помощью регистрации затухания поляризации сигнала флуоресценции, а в рамках метода поляризационной спектроскопии pump-probe - посредством регистрации линейного дихроизма зондирующего лазерного импульса, прошедшего через исследуемый молекулярный образец и задержанного относительно импульса накачки на время $t$. При этом экспериментально наблюдаемой 
величиной является зависящая от времени задержки анизотропия $r(t)$ :

$$
r(t)=\frac{I_{\|}(t)-I_{\perp}(t)}{I_{\|}(t)+2 I_{\perp}(t)},
$$

где $I_{\|}(t)$ и $I_{\perp}(t)$ - компоненты интенсивности зондирующего излучения, поглощенного исследуемыми молекулами, с поляризацией параллельной и перпендикулярной поляризации излучения накачки соответственно.

Существенным преимуществом метода накачка-зондирование по сравнению с методом флуоресценции является более высокое временное разрешение, которое позволяет исследовать сверхбыстрые процессы переноса энергии в молекулах, лежащие в субпикосекундном временном диапазоне [2]. Временная зависимость анизотропии в выражении (1) в растворах многоатомных молекул обычно определяется из эксперимента в виде сумм затухающих экспонент с весовыми коэффициентами. Однако до недавнего времени квантово-механическая теория, обосновывающая физический смысл этих экспоненциальных членов и дающая явные выражения для соответствующих весовых коэффициентов через квантово-механические матричные элементы и фазы, была развита недостаточно, что затрудняло физическую интерпретацию получающихся экспериментальных результатов.

Такая теория была построена для случая однофотонного возбуждения молекул в недавней работе авторов [21], в которой в квазиклассическом приближении было получено квантово-механическое выражение для интенсивности поглощения молекулярным образцом зондирующего импульса, задержанного относительно импульса накачки на время $t$, для произвольных состояний поляризации обоих импульсов. В рамках этой теории сигнал линейного дихроизма может быть представлен в виде вкладов от трех различных процессов: распада возбужденного состояния молекулы, вращательной диффузии и колебательной релаксации. Развитая теория была применена в работе [21] для интерпретации результатов экспериментов по возбуждению молекул биологического коэнзима $\mathrm{NADH}$ в растворах с разной вязкостью и полярностью. Актуальность подобных исследований связана с тем, что биологический коэнзим $\mathrm{NADH}$ играет важную роль в регулировании процессов клеточного метаболизма [23].

Вместе с тем интерпретация сигналов линейного дихроизма в многоатомных молекулах сталкивается с определенными неоднозначностями, связанными с наличием в многоатомных молекулах протяженных электронно-колебательных полос с большой плотностью состояний и с тем, что выстраивание осей возбужденных молекул наряду с появлением линейного дихроизма приводит также к наведенной оптической активности молекулярного образца (MO). Поэтому наряду с поглощением зондирующего излучения на резонансных электронно-колебательных переходах неизбежно возникает двулучепреломление этого излучения на близко расположенных нерезонансных переходах, причем оба эти эффекта приводят к изменению поляризации прошедшего через образец зондирующего излучения. В принципе как эффект линейного дихроизма, так и эффект двулучепреломления зондирующего излучения порознь хорошо известны в нелинейной оптике $[1,3]$. Разделение линейного дихроизма и двулучепреломления в различных молекулярных растворах исследовалось теоретически и экспериментально в работах [24-27], а выражение, описывающее вклад каждого из эффектов в сигнал для произвольного состояния поляризации накачивающего и зондирующего излучений, прошедших через атомный пар, было получено в работе [28]. Вместе с тем квантово-механические выражения, описывающие сигналы линейного дихроизма и двулучепреломления в растворах многоатомных молекул с учетом выстраивания осей молекул, возможных релаксационных процессов и когерентности колебательных состояний молекул, до настоящего времени не были известны.

Решению этой задачи посвящена настоящая работа. В ней получены выражения для изменения матрицы поляризации зондирующего излучения, прошедшего через выстроенный импульсом накачки молекулярный ансамбль, для случая произвольных поляризаций импульса излучения накачки и импульса зондирующего излучения. Вывод этих выражений основан на теории Коэн-Таннуджи и Лалое [29], в которой было получено выражение для изменения матрицы поляризации света, прошедшего через оптически ориентированный атомный пар. Настоящая работа также является обобщением подхода, основанного на методе неприводимых тензорных операторов [30,31], использованного ранее в работе [28] для описания резонансного и фарадеевского методов детектирования оптически ориентированных атомов, на случай двухступенчатого возбуждения многоатомных молекул типа асимметричного волчка в конденсированной фазе. В работе приведены и проанализированы выражения для вкладов линейного дихроизма и двулучепреломления в изменение поляризации зондирующего излучения, прошедшего через молекулярный образец, с учетом выстраивания осей молекул, возможных релаксационных процессов и когерентности колебательных состояний молекул и исследованы пути разделения этих двух вкладов в экспериментальный сигнал.

\section{2. Постановка задачи и использованный теоретический подход}

На рис. 1 схематически представлен исследуемый процесс pump-probe. В момент времени $t=0$ молекула возбуждается из основного электронного состояния $|g\rangle$ 


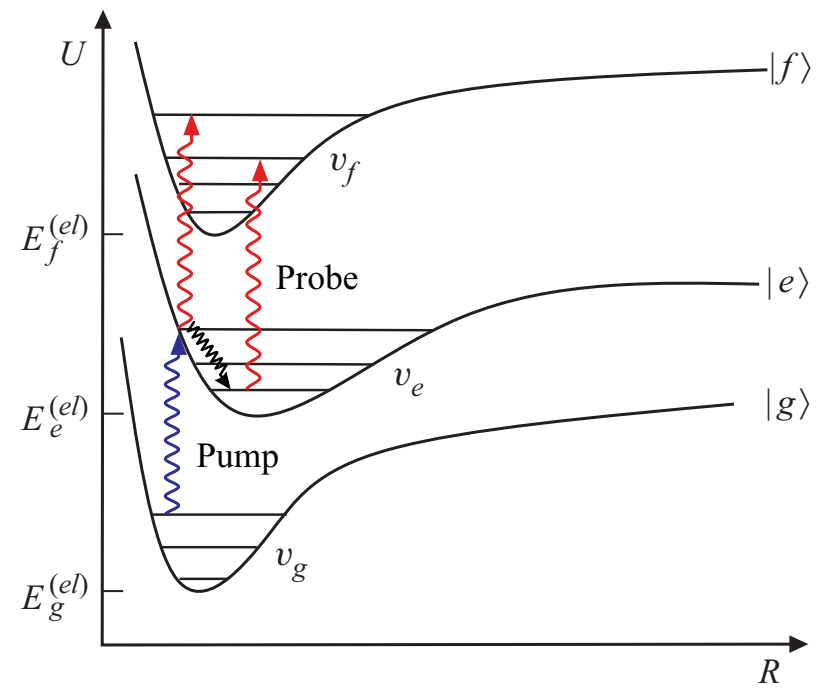

Рис. 1. Схема возбуждения многоатомной молекулы по методу pump-probe. Схематично представлены проекции поверхностей потенциальной энергии, где $|g\rangle$ соответствует основному электронному состоянию, $|e\rangle$ - возбужденному промежуточному и $|f\rangle$ - конечному. Квантовые числа $v_{g}, v_{e}$ и $v_{f}$ маркируют колебательные уровни энергии состояний $|g\rangle$, $|e\rangle$ и $|f\rangle$ соответственно.

в возбужденное электронное состояние $|e\rangle$ посредством фемтосекундного лазерного импульса накачки (pump), в результате чего заселяются высоковозбужденные колебательные уровни энергии состояния $|e\rangle$ и происходит быстрая колебательная релаксация в нижнее колебательное состояние, показанная на рис. 1 , а также другие быстрые процессы безызлучательного переноса энергии, длительность которых обычно лежит в пикосекундной или суб-пикосекундной области.

Другие процессы деградации возбужденного состояния $|e\rangle$, такие как вращательная диффузия, а также радиационный переход в основное электронное состояние, обычно характеризуются более длинными временами от сотен пикосекунд до десятков наносекунд. Изменения, происходящие в возбужденном состоянии $|e\rangle$, регистрируются с помощью зондирующего фемтосекундного импульса (probe), который задержан относительно возбуждающего импульса на время $t$ и проходит через МО, находясь либо в резонансе, либо не в резонансе с электронно-колебательными переходами из состояния $|e\rangle$ в состояние $|f\rangle$. На практике обычно регистрируется либо изменение интенсивности зондирующего излучения после прохождения молекулярного образца, либо изменение состояния его поляризации, как функции времени задержки $t$.

Таким образом, в задачи настоящей работы входили вывод и анализ выражения для изменения интенсивности и поляризации зондирующего излучения в зависимости от времени задержки $t$ от импульса накачки с учетом вышеуказанных процессов деградации возбужденного состояния.
Полные волновые функции молекулы типа асимметричного волчка для состояния $|g\rangle$ на рис. 1 в приближении Борна-Оппенгеймера могут быть записаны [32] в виде:

$$
|g\rangle=\left|n_{g}\right\rangle\left|J_{g} M_{g} k_{g}\right\rangle=\left|n_{g}\right\rangle \sum_{\Omega_{g}} A_{k_{g} \Omega_{g}}^{J_{g}}\left|J_{g} M_{g} \Omega_{g}\right\rangle,
$$

где $\left|n_{g}\right\rangle=\psi_{g}^{e l}\left|v_{g}\right\rangle, \psi_{g}^{e l},\left|v_{g}\right\rangle$ и $\left|J_{g} M_{g} k_{g}\right\rangle$ - электронная, колебательная и вращательная волновые функции молекулы, $J_{g}, M_{g}$ и $\Omega_{g}$ - полный угловой момент молекулы и его проекции на лабораторную ось $\mathrm{Z}$ и ось $\mathrm{Z}^{\prime}$ молекулы соответственно, $k_{g}$ - квантовые числа, нумерующие вращательные уровни асимметричного волчка, $A_{k_{g} \Omega_{g}}^{J_{g}}$ - коэффициенты разложения вращательной волновой функции молекулы по функциям симметричного волчка [31].

Вращательная волновая функция симметричного волчка $\left|J_{g} M_{g} \Omega_{g}\right\rangle$ в выражении 2 является нормированной $D$-функцией Вигнера [31,33]:

$$
\left|J_{g} M_{g} \Omega_{g}\right\rangle=\sqrt{\frac{2 J_{g}+1}{8 \pi^{2}}} D_{M_{g} \Omega_{g}}^{J_{g}}{ }^{*}(\phi, \theta, \xi),
$$

где $(\phi, \theta, \xi)$ - углы Эйлера, задающие главные оси инерции молекулы.

Аналогичный выражениям (2), (3) вид имеют полные волновые функции состояний $|e\rangle$ и $|f\rangle$ на рис. 1. Для описания процесса накачки и зондирования использовался формализм неприводимых тензорных операторов для матрицы плотности молекул и матрицы поляризации света. Разложение матрицы плотности вращательного движения основного состояния молекулы по ковариантным сферическим тензорам имеет вид [30,34]:

$$
\begin{gathered}
\rho_{J_{g}^{\prime} M_{g}^{\prime} k_{g}^{\prime} ; J_{g} M_{g} k_{g}}=\sum_{K Q}(-1)^{J_{g}-M_{g}} \sqrt{2 K+1} \\
\times\left(\begin{array}{ccc}
J_{g} & J_{g}^{\prime} & K \\
M_{g} & -M_{g}^{\prime} & -Q
\end{array}\right) \rho_{K Q}\left(J_{g} k_{g} J_{g}^{\prime} k_{g}^{\prime}\right),
\end{gathered}
$$

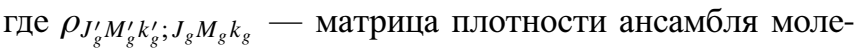
кул, $\rho_{K Q}\left(J_{g} k_{g} J_{g}^{\prime} k_{g}^{\prime}\right)-$ сферический тензор (мультиполь состояния), $K$ - ранг мультиполя состояния, принимающий значения в пределах $\left|J_{g}-J_{g}^{\prime}\right| \leq K \leq J_{g}+J_{g}^{\prime}, Q-$ компонента $K$ на ось $Z$ лабораторной системы координат $(Q=-K \ldots K)$, а выражение в круглых скобках $-3 j$ символ [33].

Мультиполь состояния ранга $K=0$ пропорционален полному количеству молекул с данным моментом $J_{g}=J_{g}^{\prime}$, мультиполи с четными значениями ранга $K$ описывают выстраивание молекул по угловым моментам $J_{g}, J_{g}^{\prime}$, а мультиполи с нечетными значениями ранга $K$ - ориентацию молекул по угловым моментам $J_{g}, J_{g}^{\prime}[30,31,35]$. При этом мультиполи с $J_{g}=J_{g}^{\prime}, k_{g}=k_{g}^{\prime}$ соответствуют диагональным по этим квантовым числам элементам матрицы плотности, а мультиполи с $J_{g} \neq J_{g}^{\prime}$ и/или $k_{g} \neq k_{g}^{\prime}$ описывают когерентности соответствующих молекулярных состояний [35]. 
Для введения матрицы поляризации света рассмотрим монохроматическое излучение с частотой $\omega$, распространяющееся вдоль оси Z. Матрица поляризации этого излучения $\pi$ определена в виде:

$$
\pi=I\left(\begin{array}{cc}
e_{\mathrm{X}} e_{\mathrm{X}}^{*} & e_{\mathrm{X}} e_{\mathrm{Y}}^{*} \\
e_{\mathrm{Y}} e_{\mathrm{X}}^{*} & e_{\mathrm{Y}} e_{\mathrm{Y}}^{*}
\end{array}\right)
$$

где $e_{\mathrm{X}}$ и $e_{\mathrm{Y}}-\mathrm{X}$ и $\mathrm{Y}$ нормированные компоненты напряженности электрического поля излучения, а $I-$ полная интенсивность излучения. Матрица $\pi$ удовлетворяет условию нормировки $\operatorname{Tr}(\pi)=I$.

Переходя в (5) от компонент напряженности электрического поля к параметрам Стокса $S_{1}, S_{2}, S_{3}$, это выражение можно представить в виде [28]:

$$
\pi=\frac{I}{2}\left(\begin{array}{cc}
1+S_{1} & S_{2}-i S_{3} \\
S_{2}+i S_{3} & 1-S_{1}
\end{array}\right),
$$

где параметр $S_{1}$ описывает линейную поляризацию излучения относительно осей $X$ и $Y$, параметр $S_{2}$ описывает линейную поляризацию излучения в системе координат, повернутой под углом $45^{\circ}$ вокруг оси Z, а параметр $S_{3}$ описывает циркулярную поляризацию.

В частности, $S_{1}=\left(I_{X}-I_{Y}\right) /\left(I_{X}+I_{Y}\right)$, где $I_{X}$ и $I_{Y}-$ интенсивности света, поляризованного по оси $X$ и $Y$ соответственно, а $S_{3}=\left(I_{+}-I_{-}\right) /\left(I_{+}+I_{-}\right)$, где $I_{ \pm}-$ интенсивности правого и левого циркулярно поляризованного света. Каждый из параметров Стокса может принимать значения в интервале от -1 до +1 .

Используя стандартные циклические компоненты $e_{q}$, где $q= \pm 1$ [33], матрицу поляризации излучения (5) можно представить в виде ковариантного неприводимого сферического тензора [31,34]:

$$
E_{K Q}(\mathbf{e})=\left[\mathbf{e} \otimes \mathbf{e}^{*}\right]_{K Q}=\sum_{q, q^{\prime}}(-1)^{q^{\prime}} e_{q} e_{q^{\prime}}^{*} C_{1 q 1-q^{\prime}}^{K Q},
$$

где член $C_{1 q 1-q^{\prime}}^{K Q}-$ коэффициент Клебша-Гордана [33], ранг $K$ ограничен значениями $K=0,1,2$, а проекция $Q$ может принимать значения $Q=-K \ldots K$.

Компонента матрицы поляризации с $K=0, Q=0$ пропорциональна интенсивности излучения, линейная поляризация излучения описывается компонентами с $K=2$, а циркулярная поляризация излучения - компонентами с $K=1$ и $K=2[30,31]$.

Переход от матриц поляризации к параметрам Стокса может быть осуществлен в соответствие с соотношениями:

$$
\begin{cases}E_{00}=-\frac{1}{\sqrt{3}}, & E_{10}=-\frac{1}{\sqrt{2}} S_{3} \\ E_{20}=-\frac{1}{\sqrt{6}}, & E_{2 \pm 2}=\frac{1}{2}\left(S_{1} \pm i S_{2}\right) .\end{cases}
$$

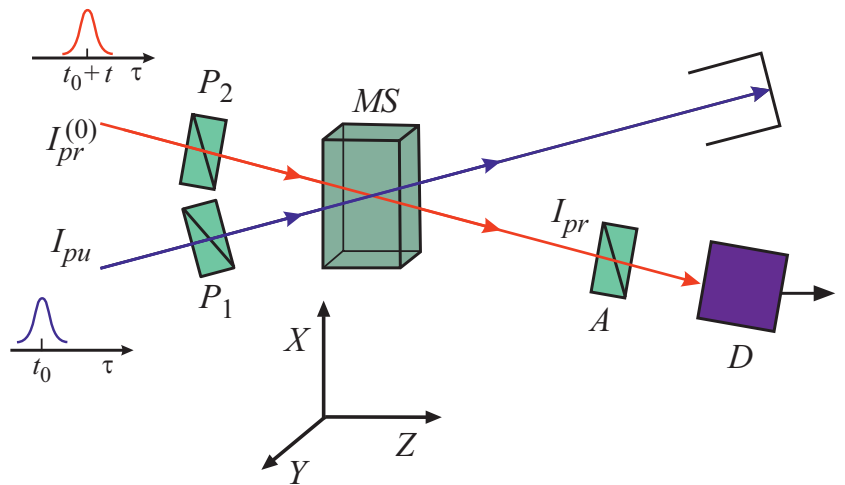

Рис. 2. Геометрия возбуждения и зондирования молекулярного образца. $I_{p u}-$ интенсивность лазерного пучка накачки. $I_{p r}^{(0)}$ и $I_{p r}$ - начальная и конечная интенсивности зондирующего лазерного пучка. $P_{1}, P_{2}-$ поляризаторы, $A-$ анализатор поляризации, $D-$ фотодетектор, $M S$ - молекулярный образец (molecular sample).

\section{3. Изменение поляризации зондирующего излучения после прохождения молекулярного образца}

Общая схема накачка-зондирование приведена на рис. 2. Согласно этой схеме, пучок накачки и пучок зондирующего излучения поляризованы произвольным образом и проходят через МО вдоль оси $\mathrm{Z}$ под малым углом друг к другу. Импульс зондирующего излучения задержан относительно импульса накачки на регулируемое время $t$, а поляризация каждого лазерного пучка независимо устанавливается с помощью поляризаторов $P_{1}$ и $P_{2}$. После прохождения $\mathrm{MO}$, зондирующее излучение пропускается через анализатор поляризации $A$ и затем поступает на фотодетектор $D$.

Для определения изменения интенсивности и поляризации зондирующего лазерного пучка на переходе $|e\rangle \rightarrow|f\rangle$ после прохождения МО рассмотрим общее выражение, описывающее изменение матрицы поляризации этого лазерного пучка: $\Delta \pi=\pi-\pi_{0}$, где $\pi_{0}-$ матрица поляризации до МO, а $\pi$ - после МО. Направления накачивающего и зондирующего лазерных пучков предполагаются почти параллельными друг другу и оси Z. Угол между этими пучками, показанный на рис. 2, значительно завышен для наглядности. В первом приближении по интенсивности излучения и для случая тонкого оптического слоя в МО это выражение может быть представлено в виде [29]:

$$
\pi-\pi_{0}=-\left[G_{A}, \pi_{0}\right]_{+}-i\left[G_{D}, \pi_{0}\right],
$$

где $[. ., . .]_{+}$- антикоммутатор и $[. ., .]-$.коммутатор.

Операторы $G_{A}$ и $G_{D}$ в $(9)$ описывают поглощение и дисперсию излучения молекулами соответственно. Оператор $G_{A}$ - эрмитов, а оператор $G_{D}-$ антиэрмитов, 
причем

$$
\begin{aligned}
G_{A} & =\frac{1}{2}\left(G+G^{\dagger}\right), \\
G_{D} & =\frac{1}{2 i}\left(G-G^{\dagger}\right) .
\end{aligned}
$$

Рассматривая распределение колебательных состояний молекулы как квазиконтинуум, циклические компоненты оператора $G$ в $(10)$ и (11) могут быть представлены в виде $[28,29]$ :

$$
\begin{aligned}
& G_{q^{\prime} q}(t)=\frac{2 \pi N L}{\hbar c} \sum_{e, e^{\prime}, f} \\
& \times \int \frac{\rho_{e e^{\prime}}(t)\left(\mathbf{e}_{q^{\prime}}^{*} \cdot \mathbf{d}_{e^{\prime} f}\right)\left(\mathbf{e}_{q} \cdot \mathbf{d}_{f e}\right) \Phi_{p r}\left(\omega-\omega_{p r}\right) \omega d \omega}{-i\left(\omega-\omega_{f e}\right)+\frac{\Gamma_{f}}{2}},
\end{aligned}
$$

где $N-$ концентрация молекул, $L-$ длина области взаимодействия лазерного излучения с $\mathrm{MO}, \hbar-$ постоянная Планка, $c$ - скорость света в вакууме, $\rho(t)$ - матрица плотности состояния $|e\rangle, \mathbf{d}$ - оператор дипольного момента молекулы, $e_{q}, e_{q^{\prime}}$, где $q, q^{\prime}= \pm 1-$ циклические компоненты поляризации, $\Phi_{p r}\left(\omega-\omega_{p r}\right)-$ спектральный профиль импульса излучения, $\omega_{p r}-$ частота максимума спектрального профиля импульса излучения, $\omega_{f e}$ - частота резонансного перехода, лежащего в пределах полосы поглощения $e \rightarrow f$, индексы $e$ и $e^{\prime}$ обозначают набор колебательных и вращательных квантовых чисел молекулы $J_{e}, M_{e}, k_{e}, v_{e}$ и $J_{e}^{\prime}, M_{e}^{\prime}, k_{e}^{\prime}, v_{e}^{\prime}$ соответственно, которые характеризуют состояние $|e\rangle$, а индексы $f-$ аналогичный набор квантовых чисел, характеризующих состояние $|f\rangle$, (рис. 1), а $\Gamma_{f}-$ скорость затухания возбужденного уровня $|f\rangle$.

В общем случае зависимость матрицы плотности $\rho_{e e^{\prime}}(t)$ в выражении (12) от времени задержки $t$ определяется релаксационными процессами, происходящими в состоянии $|e\rangle$ после импульса возбуждения в момент времени $t_{0}$, а также эффектом квантовых биений. При выводе (12) предполагалось, что длительность лазерных импульсов существенно меньше характерной длительности релаксационных процессов в состоянии $e: \tau_{i} \ll \tau_{r e l}$.

После перехода в выражениях (9)-(12) к неприводимым представлениям матрицы плотности для молекул и для зондирующего излучения по формулам (4) и (7), использования теоремы Вигнера-Эккарта и перехода в матричных элементах дипольного момента из лабораторной в молекулярную систему координат было произведено интегрирование $D$-функции Вигнера по углам Эйлера $(\phi, \theta, \xi)$ и суммирование по квантовым числам $M_{e}, M_{e}^{\prime}, J_{f}, M_{f}, k_{f}$, с использованием формул квантовой теории углового момента [33]. При этом при суммировании по квантовым числам $k_{f}$ было использовано свойство ортогональности коэффициентов разложения $A_{\Omega_{f} k_{f}}^{J_{f}}$ в (2). После ряда преобразований выражение для изменения матрицы плотности зондирующего излучения (9) после прохождения МО может быть представ- лено в виде:

$$
\begin{aligned}
& I_{p r} E_{K Q}\left(\mathbf{e}_{p r}\right)-I_{p r}^{(0)} E_{K Q}^{(0)}\left(\mathbf{e}_{p r}\right)=\frac{2 \pi N L I_{p r}^{(0)}}{\hbar c} \sum_{K_{0}, K_{e}} \sum_{\tilde{e}, \tilde{e}^{\prime}, v_{f}} \\
& \times\left[E_{K_{0}}^{(0)}\left(\mathbf{e}_{p r}\right) \otimes \rho_{K_{e}}(t)\right]_{K Q}\left\{\begin{array}{ccc}
K & K_{0} & K_{e} \\
1 & 1 & 1
\end{array}\right\} \\
& \times(-1)^{K_{0}}\left(2 K_{0}+1\right)^{1 / 2} V_{K_{e}}\left(\tilde{e}, \tilde{e}^{\prime}, v_{f}\right)\left[j_{A}\left(v_{e}, v_{f}\right)\right. \\
& \left.+(-1)^{\phi} j_{A}\left(v_{e}^{\prime}, v_{f}\right)+i\left(j_{D}\left(v_{e}, v_{f}\right)-(-1)^{\phi} j_{D}\left(v_{e}^{\prime}, v_{f}\right)\right)\right],
\end{aligned}
$$

где $I_{p r}^{(0)}, I_{p r}-$ интенсивности падающего и прошедшего излучения соответственно, $\mathbf{e}_{p r}-$ вектор поляризации зондирующего излучения, $E_{K Q}\left(\mathbf{e}_{p r}\right)-$ матрица поляризации прошедшего через МО зондирующего излучения, $E_{K Q}^{(0)}\left(\mathbf{e}_{p r}\right)-$ матрица поляризации исходного зондирующего излучения, выражение в фигурных скобках $-6 j$ символ [33], $\phi=K_{0}+K_{e}+K$, индексы $K, Q$ относятся к прошедшему зондирующему излучению, а $K_{0}, Q_{0}-$ к падающему.

Индексы суммирования $\tilde{e}$ и $\tilde{e}^{\prime}$ в выражении (13) обозначают наборы квантовых числел $J_{e}, k_{e}, v_{e}$ и $J_{e}^{\prime}, k_{e}^{\prime}, v_{e}^{\prime}$ соответственно, характеризующие возбужденное состояние $|e\rangle$. Член в квадратных скобках в первой строке является неприводимым тензорным произведением [33] матрицы плотности излучения $E_{K_{0} Q_{0}}^{(0)}\left(\mathbf{e}_{p r}\right)$ и мультиполя состояния $\rho_{K_{e} Q_{e}}(t)$ молекулярного ансамбля (4) в состоянии $|e\rangle$, который зависит также от квантовых чисел колебательных и вращательных состояний $e$.

Величина $V_{K_{e}}\left(\tilde{e}, \tilde{e}^{\prime}, v_{f}\right)$ в (13) содержит в себе матричные элементы проекций операторов дипольного момента молекулы:

$$
\begin{aligned}
& V_{K_{e}}\left(\tilde{e}, \tilde{e}^{\prime}, v_{f}\right)=(-1)^{J_{e}^{\prime}+J_{e}+1}\left[\left(2 K_{e}+1\right)\left(2 J_{e}^{\prime}+1\right)\right]^{1 / 2} \\
& \times 3^{-1 / 2} \sum_{\Omega_{e}, \Omega_{e}^{\prime}} A_{\Omega_{e}}^{J_{e}^{*}} A_{e}^{J_{\Omega_{e}^{\prime}}^{\prime}} A_{\Omega_{e}^{\prime} k_{e}^{\prime}} \sum_{q_{2}, q_{2}^{\prime}} \sum_{n_{f}, \tilde{q}}\left\langle n_{f}\left|d_{q_{2}}\right| n_{e}\right\rangle^{*}\left\langle n_{f}\left|d_{q_{2}^{\prime}}\right| n_{e}^{\prime}\right\rangle \\
& \times C_{K_{e} \tilde{q} 1 q_{2}}^{1 q_{2}^{\prime}} C_{K_{e}}^{J_{e} \Omega_{e} \Omega_{e} J_{e}^{\prime} \Omega_{e}^{\prime},}
\end{aligned}
$$

где величины $d_{q_{2}}, d_{q_{2}^{\prime}}$ обозначают циклические компоненты оператора дипольного момента молекулы относительно молекулярной системы координат $\mathrm{X}^{\prime} \mathrm{Y}^{\prime} \mathrm{Z}^{\prime}$.

Слагаемые в (13), содержащие члены $j_{A}\left(v_{e}, v_{f}\right)$ и $j_{D}\left(v_{e}, v_{f}\right)$, связаны с поглощением и дисперсией зондирующего излучения в молекулярной системе соответственно, они представляют собой интегралы вида:

$$
\begin{gathered}
j_{A}\left(v_{e}, v_{f}\right)=\int \frac{\Phi_{p r}\left(\omega-\omega_{p r}\right) \frac{\Gamma_{f}}{2} \omega d \omega}{\left(\omega-\omega_{f e}\right)^{2}+\frac{\Gamma_{f}^{2}}{4}}, \\
j_{D}\left(v_{e}, v_{f}\right)=\int \frac{\Phi_{p r}\left(\omega-\omega_{p r}\right)\left(\omega-\omega_{f e}\right) \omega d \omega}{\left(\omega-\omega_{f e}\right)^{2}+\frac{\Gamma_{f}^{2}}{4}} .
\end{gathered}
$$


Частота $\omega_{f e}$ в (15), (16) может быть представлена как сумма чисто электронной и чисто колебательной составляющих: $\omega_{f e}=\omega_{f e}^{(e l)}+\omega_{f e}^{(v)}$, причем $\hbar \omega_{f e}^{(e l)}=\left(E_{f}^{(e l)}-E_{e}^{(e l)}\right)$, а $\hbar \omega_{f e}^{(v)}=\left(E\left(v_{f}\right)-E_{f}^{(e l)}\right)-$ $-\left(E\left(v_{e}\right)-E_{e}^{(e l)}\right)$, где $E\left(v_{f}\right), E\left(v_{e}\right)$ и $E_{f, e}^{(e l)}$, соответствующие полные энергии электроно-колебательных уровней молекулы и чисто электронные энергии состояний $|f\rangle$ и $|e\rangle$. Таким образом, выражение (13) описывает изменения интенсивности и поляризации зондирующего лазерного пучка на переходе $|e\rangle \rightarrow|f\rangle$, показанном на рис. 1, в зависимости от матрицы плотности возбужденного состояния $\rho_{K_{e} Q_{e}}(t)$.

Для определения явного вида мультиполя состояния $\rho_{K_{e} Q_{e}}(t)$ рассмотрим возбуждение состояния $|e\rangle$ коротким лазерным импульсом накачки из основного состояния молекулы $|g\rangle$ как изображено на рис. 1. Предполагая, что основное состояние молекулы не поляризовано и его мультиполь состояния полностью характеризуется рангом $K_{g}=0$, уравнение эволюции для $\rho_{K_{e} Q_{e}}(t)$ в пределе, когда длительность импульса накачки значительно короче времен релаксации и единственными источниками релаксации состояния $|e\rangle$ являются его спонтанный радиационный распад и изотропные безызлучательные взаимодействия, можно записать в виде [35]:

$$
\frac{d \rho_{K_{e} Q_{e}}(t)}{d t}=F_{K_{e} Q_{e}} \delta(t)-i \omega_{e e^{\prime}} \rho_{K_{e} Q_{e}}(t)-\frac{\rho_{K_{e} Q_{e}}(t)}{T},
$$

где $T=1 / \Gamma_{e}-$ время жизни состояния $|e\rangle, \omega_{e e^{\prime}}=\omega_{e}-\omega_{e}^{\prime}$, а $F_{K_{e} Q_{e}}=\rho_{K_{e} Q_{e}}(0)$ - матрица накачки, которая для случая возбуждения произвольной многоатомной молекулы после ряда преобразований квантовой теории углового момента может быть представлена в виде:

$$
\begin{aligned}
& F_{K_{e} Q_{e}}=F_{0} E_{K_{e} Q_{e}}\left(\mathbf{e}_{p u}\right) \sum_{J_{g}, \Omega_{g}} \sum_{\Omega_{e} \Omega_{e}^{\prime}}(-1)^{J_{g}+J_{e}} A_{\Omega_{e} k_{e}}^{J_{e}} A_{\Omega_{e}^{\prime} k_{e}^{\prime}}^{J_{e}^{\prime *}} \\
& \quad \times\left\{\begin{array}{ccc}
1 & 1 & K_{e} \\
J_{e}^{\prime} & J_{e} & J_{g}
\end{array}\right\} \sum_{v_{g}} R^{p u}\left(J_{g} \Omega_{g} v_{g}\right) \sum_{q_{1}, q_{1}^{\prime}} C_{J_{g} \Omega_{g} \Omega_{2} \Omega_{1}}^{J_{e}} \\
& \quad \times C_{J_{g} \Omega_{g} 1 q_{1}^{\prime}}^{J^{\prime} \Omega_{e}^{\prime}}\left\langle\psi_{e}^{e l}\left|d_{q_{1}}\right| \psi_{g}^{e l}\right\rangle^{*}\left\langle\psi_{e}^{e l}\left|d_{q_{1}^{\prime}}\right| \psi_{g}^{e l}\right\rangle,
\end{aligned}
$$

где $F_{0}-$ константа, пропорциональная интенсивности излучения накачки, $E_{K_{e} Q_{e}}\left(\mathbf{e}_{p u}\right)-$ матрица поляризации излучения накачки, а индексы $q_{1}, q_{1}^{\prime}$ обозначают циклические проекции оператора дипольного момента молекулы на ее ось.

Функция $R^{p u}\left(J_{g} \Omega_{g} v_{g}\right)$ в выражении (18) равна [35]:

$$
\begin{aligned}
& R^{p u}\left(J_{g} \Omega_{g} v_{g}\right)=\left\langle v_{e}^{\prime} \mid v_{g}\right\rangle\left\langle v_{e} \mid v_{g}\right\rangle^{*} \\
& \quad \times \int \frac{i \Phi_{p u}\left(\omega-\omega_{p u}\right)\left(\omega_{e e^{\prime}}+i \Gamma_{e}\right) d \omega}{\left(\omega_{e^{\prime} g}-\omega+i \Gamma_{e} / 2\right)\left(\omega_{e g}-\omega-i \Gamma_{e} / 2\right)} \\
& \quad \times \sum_{k_{g}}\left|A_{\Omega_{g} k_{g}}^{J_{g}}\right|^{2} N_{J_{g}, k_{g}, v_{g}},
\end{aligned}
$$

где $\Phi_{p u}\left(\omega-\omega_{p u}\right)$ - спектральный профиль импульса накачки, $\omega_{e g}=\omega_{e}-\omega_{g}$, а $N_{J_{g}, k_{g}, v_{g}}-$ распределение молекул по вращательным и колебательным уровням энергии основного состоянии $|g\rangle$.

Величины $\left\langle v_{e}^{\prime} \mid v_{g}\right\rangle$ в выражении (19) представляют собой интергалы перекрытия колебательных волновых функций молекулы (интегралы Франка-Кондона [36]).

Отметим, что если матричные элементы дипольного момента в (18) записаны в системе координат накачки, в которой молекулярная ось $Z^{\prime}$ направлена вдоль вектора дипольного момента перехода $\mathbf{d}^{p u}$, то оба индекса $q_{1}$ и $q_{1}^{\prime}$ равны нулю. При этом единственная отличная от нуля проекция вектора $\mathbf{d}^{p u}$ равна $d_{0}^{p u}=d_{Z}^{p u}$. Однако при этом индексы $q_{2}$ и $q_{2}^{\prime}$ в выражении (14) в системе координат „накачки“ могут быть отличны от нуля, поскольку в общем случае векторы дипольных моментов $\mathbf{d}^{p u}$ и $\mathbf{d}^{p r}$ не параллельны друг другу. Предполагая, что симметрия молекулы мало отличается от симметричного волчка, положение вектора дипольного момента $\mathbf{d}^{p r}$ относительно вектора $\mathbf{d}^{p u}$ можно охарактеризовать одним углом Эйлера $\theta$, который в общем случае зависит от энергии перехода в полосе $e \rightarrow f$, т. е. от колебательных квантовых чисел $v_{e}$ и $v_{f}$. Тогда компоненты вектора дипольного момента на переходе $|e\rangle \rightarrow|f\rangle$ можно записать в виде:

$$
d_{q_{2}}^{p r}=D_{q_{2} 0}^{1^{*}}(0, \theta, 0) d_{0}^{p r},
$$

где $d_{0}^{p r}=d_{Z}^{p r}$ - проекция дипольного момента на ось $Z^{\prime \prime}$ в системе координат „зондирования“", в которой ось $Z^{\prime \prime}$ направлена вдоль вектора дипольного момента перехода $\mathbf{d}^{p r}$, а $D_{q_{2} 0}^{1^{*}}(0, \theta, 0)-D$-функция Вигнера.

Переходя в матричных элементах дипольного момента в выражении (14) в систему координат „зондирования“", сумма по двум $D$-функциям Вигнера и коэффициенту Клебша-Гордана для случая изотропных взаимодействий с молекулами растворителя может быть представлена в виде [22,34]:

$$
\begin{gathered}
\sum_{q_{2}, q_{2}^{\prime}} D_{q_{2} 0}^{1^{*}}(0, \theta, 0) D_{q_{2}^{\prime} 0}^{1}(0, \theta, 0) C_{K_{e} \tilde{q} 1 q_{2}}^{1 q_{2}^{\prime}} \\
\quad=\delta_{\tilde{q}, 0} C_{10 K_{e} 0}^{10} P_{K_{e}}(\cos \theta),
\end{gathered}
$$

где $P_{K_{e}}(\cos \theta)$ - полином Лежандра ранга $K_{e}$, который может принимать только значения $K_{e}=0,2$.

Решение уравнения (17) имеет простой вид:

$$
\rho_{K_{e} Q_{e}}(t)=F_{K_{e} Q_{e}} e^{-i \omega_{e e^{\prime}} t-t / T} .
$$

Однако необходимо отметить, что выражение (22) не учитывает эффекты анизотропной релаксации состояния $|e\rangle$, которые часто необходимо принимать во внимание при исследовании эволюции возбужденных молекул в конденсированной фазе. К таким эффектам, в частности, относятся вращательная диффузия и анизотропная колебательная релаксация [21]. Вводя оба эти эффекта в выражения (13), (14), (18), (19) по методике, изложенной в работах [21,34], с учетом выражения (21) и проводя суммирование по вращательным квантовым числам промежуточных состояний $J_{e}, J_{e}^{\prime}, \Omega_{e}, \Omega_{e}^{\prime}, k_{e}, k_{e}^{\prime}$ 
с использованием свойства ортогональности коэффициентов разложения $A_{\Omega_{e} k_{e}}^{J_{e}}$, с помощью алгебры квантовой теории угловых моментов [33] выражение для изменения матрицы поляризации зондирующего излучения (13) можно представить в следующем виде:

$$
\begin{aligned}
& I_{p r} E_{K Q}\left(\mathbf{e}_{p r}\right)-I_{p r}^{(0)} E_{K Q}^{(0)}\left(\mathbf{e}_{p r}\right)=C I_{p r}^{(0)} e^{-t / T} \sum_{K_{0}, K_{e}} \sum_{v_{e}, v_{e}^{\prime}} \\
& \times\left[E_{K_{0}}^{(0)}\left(\mathbf{e}_{p r}\right) \otimes E_{K_{e}}\left(\mathbf{e}_{p u}\right)\right]_{K Q} R^{p u} M_{K_{e}}^{p r} \rho_{v_{e} v_{e}^{\prime}}(t) D_{00}^{K_{e}}(t) \\
& \times(-1)^{K_{0}+K_{e}}\left[\left(2 K_{0}+1\right)\left(2 K_{e}+1\right)\right]^{1 / 2}\left[C_{10}^{10} K_{e} 0\right]^{2} \\
& \times\left\{\begin{array}{ccc}
K & K_{0} & K_{e} \\
1 & 1 & 1
\end{array}\right\}\left[j_{A}\left(v_{e}, v_{f}\right)+(-1)^{\phi} j_{A}\left(v_{e}^{\prime}, v_{f}\right)\right. \\
& \left.+i\left(j_{D}\left(v_{e}, v_{f}\right)-(-1)^{\phi} j_{D}\left(v_{e}^{\prime}, v_{f}\right)\right)\right],
\end{aligned}
$$

где фаза $\phi=K+K_{e}+K_{0}$, а

$$
\begin{array}{r}
M_{K_{e}}^{p r}=\sum_{v_{f}}\left\langle v_{f} \mid v_{e}^{\prime}\right\rangle\left\langle v_{f} \mid v_{e}\right\rangle^{*} P_{K_{e}}(\cos \theta), \\
R^{p u}=\sum_{v_{g}} \sum_{J_{g}, \Omega_{g}}\left(2 J_{g}+1\right) R^{(p u)}\left(J_{g} \Omega_{g} v_{g}\right), \\
C=\frac{6 \pi N L F_{0}}{\hbar c}\left|\left\langle\psi_{f}^{e l}\left|d_{Z},\right| \psi_{e}^{e l}\right\rangle\right|^{2}\left|\left\langle\psi_{e}^{e l}\left|d_{Z}{ }^{\prime}\right| \psi_{g}^{e l}\right\rangle\right|^{2},
\end{array}
$$

где функция $R^{(p u)}\left(J_{g} \Omega_{g} v_{g}\right)$ определена в (19).

Матрица плотности $\rho_{v_{e} v_{e}^{\prime}}(t)$ в выражении (23) описывает колебательные и релаксационные процессы в квантовых состояниях $v_{e}, v_{e}^{\prime}$ после их возбуждения из основного состояния $|g\rangle$ коротким лазерным импульсом накачки, а член $D_{00}^{K_{e}}(t)$ описывает вращательную диффузию в возбужденном состоянии $|e\rangle$ [21]. В приближении квантовой теории релаксации временная зависимость матрицы плотности колебательных состояний $\rho_{v_{e} v_{e}^{\prime}}(t)$ может быть представлена в виде: $\rho_{v_{e} v_{e}^{\prime}}(t) \sim \exp \left(-i \omega_{e, e^{\prime}} t-t / \tau_{v}\right)$, где осциллирующая на частоте $\omega_{e^{\prime} e}$ экспонента, содержащая разность частот колебательных состояний $v_{e}$ и $v_{e}^{\prime}$, описывает известный эффект квантовых биений [35], а диагональные элементы с $v_{e}=v_{e}^{\prime}-$ изменение населенности колебательных состояний вследствие быстрой колебательной релаксации [21]. В условиях большой плотности колебательных состояний в основном и возбужденных состояниях многоатомных молекул, усреднение осциллирующих элементов матрицы плотности в (23) после интегрирования по частотам $\omega_{e, e^{\prime}}=\omega_{e f}-\omega_{e^{\prime} f}$ в пределах широких полос возбуждения приводит к эффективному подавлению вклада недиагональных элементов матрицы плотности по сравнению с вкладом диагональных элементов. Поэтому в дальнейшем будет рассматриваться только вклад от диагональных элементов матрицы плотности $\rho_{v_{e} v_{e}^{\prime}}(t)$, где $v_{e}=v_{e}^{\prime}$.

\section{4. Анализ полученных результатов}

Полученные выражения (23)-(26) описывают изменение матрицы поляризации и интенсивности зондиру- ющего импульса излучения $I_{p r} E_{K Q}\left(\mathbf{e}_{p r}\right)-I_{p r}^{(0)} E_{K Q}^{(0)}\left(\mathbf{e}_{p r}\right)$ в зависимости от матрицы поляризации импульса накачки $E_{K_{e} Q_{e}}\left(\mathbf{e}_{p u}\right)$ и от структуры и динамики возбужденных состояний произвольной многоатомной молекулы, схематически изображенных на рис. 1. В частности, выражение (23) связывает поляризацию и интенсивность прошедшего через МО зондирующего импульса с поляризацией импульсов накачки и зондирования и содержит информацию о дихроизме и двулучепреломлении зондирующего излучения в МО в зависимости от когерентности колебательных состояний $v_{e}, v_{e}^{\prime}$ и от значения угла поворота дипольного момента перехода $\theta$, а также о динамических процессах в возбужденном состоянии молекулы $|e\rangle$, связанных с радиационными переходами и релаксацией колебательных и вращательных состояний.

Ранг излучения импульса накачки $K_{e}$ в (23) может принимать только значения $K_{e}=0$ и 2 вследствие свойства симметрии коэффициента Клебша-Гордана $C_{10}^{10} K_{e}$. Поэтому в рассмотренных условиях любая поляризация импульса накачки может приводить только к выстраиванию осей возбуждаемых молекул, но не к их ориентации.

Сомножитель в квадратных скобках в четвертой строке выражения (23) описывает изменение интенсивности и поляризации зондирующего излучения вследствие дихроизма и двулучепреломления МО, определяемых функциями $j_{A}(15)$ и $j_{D}(16)$. Как видно, вклады от этих эффектов зависят от значения фазового множителя $\phi=\left(K_{0}+K_{e}+K\right)$. В общем случае при наличии когерентности между колебательными состояниями молекулы (т.е. при $v_{e} \neq v_{e}^{\prime}$ ) члены $j_{A}$ и $j_{D}$ в выражении (23) не разделяются при любой поляризации зондирующего и накачивающего излучений, т.е. наблюдаемый сигнал содержит вклады как от эффекта дихроизма, так и двулучепреломления.

В случае $v_{e}=v_{e}^{\prime}$ член в квадратных скобках в четвертой строке выражения (23) преобразуется к виду:

$$
\begin{aligned}
& {\left[j_{A}\left(v_{e}, v_{f}\right)+(-1)^{\phi} j_{A}\left(v_{e}^{\prime}, v_{f}\right)+i\left(j_{D}\left(v_{e}, v_{f}\right)\right.\right.} \\
& \left.\left.\quad-(-1)^{\phi} j_{D}\left(v_{e}^{\prime}, v_{f}\right)\right)\right]=\left[j_{A}\left(v_{e}, v_{f}\right)\left(1+(-1)^{\phi}\right)\right. \\
& \left.\quad+i j_{D}\left(v_{e}, v_{f}\right)\left(1-(-1)^{\phi}\right)\right] .
\end{aligned}
$$

Как видно из выражения (27), эффект дихроизма может быть зарегистрирован только при условии, что сумма $\left(K+K_{0}\right)$ в $(23)$ имеет четное значение, а эффект двулучепреломления - только если эта сумма является нечетной, что, как будет показано ниже, позволяет экспериментально разделить вклады в сигнал от двух этих эффектов. Полученный результат совпадает с выводами работы [28], в которой рассматривалось детектирование дихроизма и двулучепреломления в парах поляризованных атомов. Можно также показать, что при учете только некогерентных членов $v_{e}=v_{e}^{\prime}$ часть выражения (23), описывающая линейный дихроизм зондирующего излучения при прохождении МО, эквивалентна выражению, полученному в нашей недавней работе [21]. 
Рассмотрим экспериментальную геометрию, использованную во многих работах при наблюдении оптического эффекта Керра и линейного дихроизма [3,25-27], когда излучение накачки линейно поляризовано вдоль оси $\mathrm{X}$ (рис. 2), а зондирующее излучение первоначально поляризовано линейно под углом $45^{\circ}$ к оси $\mathrm{X}$, причем перед фотодетектором установлен анализатор поляризации $A$. При этом, поскольку для линейно поляризованного света компонента поляризационной матрицы (7) ранга $K_{0}=1$ равна нулю [35], то регистрация линейного дихроизма возможна только если ранг $K=2$, т.е. если анализатор $A$ на рис. 2 выделяет линейную компоненту поляризации прошедшего зондирующего излучения. При этом регистрация двулучепреломления возможна только если ранг $K=1$, т.е. если анализатор $A$ на рис. 2 выделяет циркулярную компоненту поляризации прошедшего зондирующего излучения. Переходя от поляризаций излучения к параметрам Стокса (6), можно утверждать, что для наблюдения линейного дихроизма зондирующего излучения анализатор $A$ должен быть настроен на регистрацию параметра Стокса $S_{1}$, а для наблюдения двулучепреломления зондирующего излучения анализатор $A$ должен быть настроен на регистрацию параметра Стокса $S_{3}$. Переход в выражении (23) от матриц поляризации к параметрам Стокса может быть осуществлен в соответствие с соотношениями (8).

Необходимо отметить, что сдвиги фаз волнового фронта, вносимые любыми элементами оптического тракта регистрирующего излучения, будут неизбежно влиять на правильную настройку анализатора $A$. При этом, чтобы избежать наложения сигналов дихроизма и двулучепреломления, сдвиг фаз, вносимый анализатором $A$ в проходящее излучение, должен учитывать также сдвиги фаз, вносимые всеми другими оптическими элементами. Необходимость такой фазовой компенсации ранее в литературе не обсуждалась.

Отметим, что частота резонанса $\omega_{f e}$ в выражениях (15), (16) зависит как от электронных квантовых чисел $e, f$, так и от колебательных квантовых чисел $v_{e}, v_{f}$ в (24). Поэтому при вычислении сигналов линейного дихроизма и двулучепреломления на основе выражения (23) с учетом интегралов (15), (16) необходимо провести усреднения по полосе поглощения молекулы, обусловленное суммированием по всем возможным колебательным состояниям $v_{e}$ и $v_{f}$ с учетом соответствующих интегралов Франка-Кондона и по частоте $\omega$ в пределах спектра возбуждения. Учитывая, что колебательные уровни энергии в многоатомных молекулах образуют квазиконтинуум, суммирование по $v_{e}, v_{f}$ можно заменить интегрированием. Вводя аналитическую функцию $F\left(\omega_{f e}-\omega_{0}\right)$, описывающую распределение частот в полосе поглощения молекулы на переходе $|e\rangle \rightarrow|f\rangle$, сигналы линейного дихроизма и двулучепреломления могут быть представлены в виде:

$$
I_{A}\left(\omega_{p r}\right) \sim \iint \frac{F\left(\omega_{f e}-\omega_{0}\right) \Phi_{p r}\left(\omega-\omega_{p r}\right) \frac{\Gamma}{2} \omega d \omega d \omega_{f e}}{\left(\omega-\omega_{f e}\right)^{2}+\frac{\Gamma^{2}}{4}}
$$

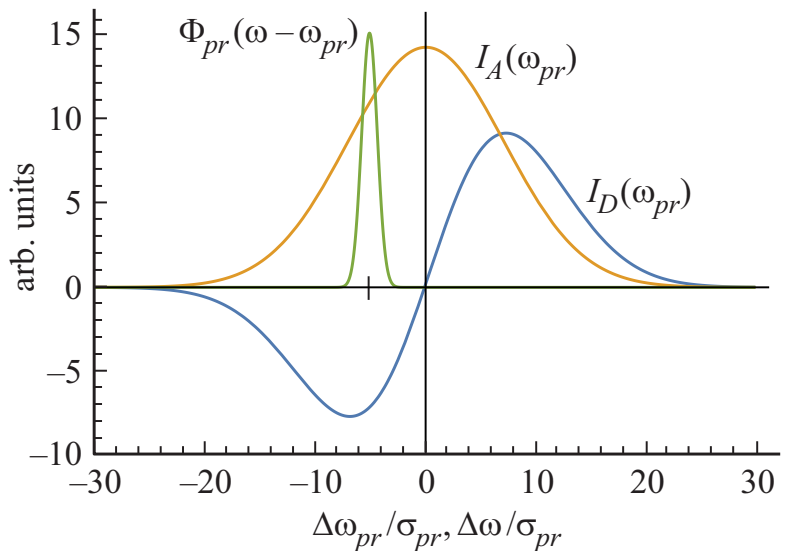

Рис. 3. Спектральные зависимости сигналов линейного дихроизма и двулучепреломлания для многоатомной молекулы. $I_{A}\left(\omega_{p r}\right)$ - сигнал линейного дихроизма $(28)$ в зависимости от приведенной частоты максимума спектра зондирующего излучения $\Delta \omega_{p r} / \sigma_{p r} ; I_{D}\left(\omega_{p r}\right)$ - сигнал двулучепреломления (29) в зависимости от приведенной частоты максимума спектра зондирующего излучения $\Delta \omega_{p r} / \sigma_{p r} ; \Phi\left(\omega-\omega_{p r}\right)-$ спектральный профиль зондирующего импульса в зависимости от приведенной частоты зондирующего излучения $\Delta \omega / \sigma_{p r}$; $\Delta \omega_{p r}=\left(\omega_{p r}-\omega_{\max }\right) ; \Delta \omega=\left(\omega-\omega_{p r}\right) ; \sigma_{p r}$ - дисперсия функции спектрального распределения зондирующего импульса; $\omega_{\max }$ - частота максимума кривой $I_{A}\left(\omega_{p r}\right)$.

$$
I_{D}\left(\omega_{p r}\right) \sim \iint \frac{F\left(\omega_{f e}-\omega_{0}\right) \Phi_{p r}\left(\omega-\omega_{p r}\right)\left(\omega-\omega_{f e}\right) \omega d \omega d \omega_{f e}}{\left(\omega-\omega_{f e}\right)^{2}+\frac{\Gamma^{2}}{4}}
$$

Для уточнения физического смысла выражений (28) и (29) была использована модель, в рамках которой спектр возбуждения $\Phi_{p r}\left(\omega-\omega_{p r}\right)$ и распределение колебательных частот молекулы на переходе $e \rightarrow f$ $F\left(\omega_{f e}-\omega_{0}\right)$ были представлены в виде гауссовых функций с максимумами $\omega_{p r}$ и $\omega_{0}$ и дисперсиями $\sigma_{p r}$ и $\sigma_{0}$ соответственно. Затем в выражениях (28) и (29) было произведено интегрирование по переменным $\omega, \omega_{f e}$. В расчете было использовано ориентировочное значение спектральной ширины поглощения в $10^{14} \mathrm{~s}^{-1}$, характерной для водного раствора молекул $\mathrm{NADH}$, и ширины спектрального контура возбуждения около $10^{13} \mathrm{~s}^{-1}$, характеризующей фемтосекундные импульсы, использованные в работе [21]. Отметим, что константа затухания $\Gamma$ в (15), (16) имеет величину порядка $10^{9} \mathrm{~s}^{-1}$, поэтому характерная ширина соответствующего резонансного контура на несколько порядков меньше, чем ширина как спектрального контура лазерного импульса $\Phi\left(\omega-\omega_{p r}\right)$, так и спектра полосы молекулярного поглощения $F\left(\omega_{f e}-\omega_{0}\right)$. Поэтому знаменатель в выражении (28) представляет собой, по-существу, $\delta$-функцию и интеграл по $\omega_{f e}$ легко берется.

Полученные спектральные зависимости сигналов дихроизма $I_{A}$ и двулучепреломления $I_{D}$ представлены на рис. 3 в зависимости от приведенной расстройки $\Delta \omega_{p r} / \sigma_{p r}$, где $\Delta \omega_{p r}=\left(\omega_{p r}-\omega_{\max }\right)$ между максимумом волнового пакета зондирующего импульса $\omega_{p r}$ и мак- 
симумом полосы поглощения молекулы на переходе $|e\rangle \rightarrow|f\rangle, \omega_{\max }$. На том же рисунке показан спектральный контур лазерного импульса $\Phi\left(\omega-\omega_{p r}\right)$ в зависимости от частоты приведенной расстройки $\Delta \omega / \sigma_{p r}$, где $\Delta \omega=\left(\omega-\omega_{p r}\right)$. Как видно из рис. 3 , сигнал дихроизма $I_{A}$ имеет вид кривой поглощения, а сигнал двулучепреломления $I_{D}$ - кривой дисперсии. Поэтому спектральные области наблюдения обоих сигналов заметно перекрываются, что подтверждает актуальность задачи их раздельной регистрации, рассмотренной в настоящей работе.

Рассмотрим разделение вкладов сигналов линейного дихроизма и двулучепреломления в (23) для случая поляризационно-модуляционной схемы эксперимента, реализованной в наших недавних работах [20,21]. В этих работах лазерные пучки накачки и зондирования распространялись вдоль оси Z, как показано на рис. 2. Поляризация цуга импульсов излучения накачки была промодулирована на частоте $100 \mathrm{kHz}$ с помощью акустооптического модулятора, причем поляризация осциллировала между вертикальным (вдоль оси X) и горизонтальным (вдоль оси Y) направлениями. Зондирующее излучение было первоначально поляризовано под углом $45^{\circ}$ к оси $\mathrm{X}$, а после прохождения МО разность его поляризационных компонент $I_{\mathrm{X}}-I_{\mathrm{Y}}$ регистрировалась с помощью фотодетектора и полученный электрический сигнал на частоте модуляции $\Omega=100 \mathrm{kHz}$ выделялся узкополосным синхронным детектором. Отметим, что частота следования лазерных импульсов накачки в эксперименте $[20,21] f_{p}=80 \mathrm{MHz}$ существенно превышала частоту модуляции поляризации $100 \mathrm{kHz}$, поэтому пучки накачки и зондирования могли рассматриваться как квазинепрерывные.

Используя явный вид матриц поляризации накачки и зондирования, а также пользуясь формулой (23), выпишем полную интенсивность прошедшего излучения и связанные с ним параметры Стокса. Предполагая, что колебания акусто-оптического модулятора осуществляются на частототе $\Omega / 2$ по закону $\sin \left(\frac{\Omega}{2} t\right)$, колебания вектора поляризация излучения накачки могут быть описаны выражением:

$$
\mathbf{e}_{p u}(t)=\frac{1}{2}\left[\mathbf{e}_{\mathrm{X}}\left(1+e^{i \varphi(t)}\right)+\mathbf{e}_{\mathrm{Y}}\left(1-e^{i \varphi(t)}\right)\right],
$$

где фаза $\varphi(t)=\pi \sin \left(\frac{\Omega}{2} t\right)$, а циклические компоненты поляризации можно записать в виде:

$$
\begin{aligned}
& \mathbf{e}_{1}(t)=-\frac{1}{2 \sqrt{2}}\left[\mathbf{e}_{\mathrm{X}}\left(1+e^{i \varphi(t)}\right)+i \mathbf{e}_{\mathrm{Y}}\left(1-e^{i \varphi(t)}\right)\right], \\
& \mathbf{e}_{-1}(t)=\frac{1}{2 \sqrt{2}}\left[\mathbf{e}_{\mathrm{X}}\left(1+e^{i \varphi(t)}\right)-i \mathbf{e}_{\mathrm{Y}}\left(1-e^{i \varphi(t)}\right)\right] .
\end{aligned}
$$

При этом отличные от нуля компоненты матрицы поляризации излучения накачки и зондирования (7) равны:

$$
E_{00}\left(\mathbf{e}_{p u}\right)=-\frac{1}{\sqrt{3}}, \quad E_{00}^{(0)}\left(\mathbf{e}_{p r}\right)=-\frac{1}{\sqrt{3}},
$$

$$
\begin{array}{r}
E_{20}\left(\mathbf{e}_{p u}\right)=-\frac{1}{\sqrt{6}}, \quad E_{20}^{(0)}\left(\mathbf{e}_{p r}\right)=-\frac{1}{\sqrt{6}}, \\
E_{2 \pm 2}\left(\mathbf{e}_{p u}\right)=\frac{1}{2} \cos \varphi(t), \quad E_{2 \pm 2}^{(0)}\left(\mathbf{e}_{p r}\right)= \pm \frac{i}{2} .
\end{array}
$$

Как видно из выражений (33)-(35), только компоненты матрицы поляризации излучения накачки $E_{22}$ и $E_{2-2}$ модулированы во времени и могут вносить вклад в наблюдаемые экспериментальные сигналы.

Подставляя компоненты матриц поляризации из (33)-(35) в выражение (23) и фиксируя только компоненту прошедшего зондирующего излучения $E_{00}\left(\mathbf{e}_{p r}\right)=-1 / \sqrt{3}$, выражение для полной интенсивности цуга импульсов прошедшего через MO зондирующего пучка $I_{p r}=I_{\mathrm{X}}+I_{\max }$ может быть записано в виде:

$$
I_{p r}=I_{p r}^{(0)} \sum_{n}\left[\theta\left(t_{n}\right)-\theta\left(t_{n+1}\right)\right]\left(1-C e^{-\frac{t_{n}}{T}}\left(2 R_{0}^{p r}\left(t_{n}\right)+r_{A}\left(t_{n}\right)\right)\right),
$$

где суммирование проводится по $n$ импульсам зондирования от $n=0$ до $n=N \gg 1, T_{p}$ - период повторения импульсов, $t_{n}=t-n T_{p}$, константа $C$ определена в выражении (26), а $\theta(x)$ - единичная ступенчатая функция Хевисайда.

В выражении (36) анизотропия $r_{A}\left(t_{n}\right)$ равна

$$
r_{A}\left(t_{n}\right)=\frac{2}{5} R_{2}^{p r(A)}\left(t_{n}\right) D_{00}^{2}\left(t_{n}\right),
$$

a

$$
R_{K_{e}}^{p r(A)}\left(t_{n}\right)=\frac{1}{3} \sum_{v_{e}, v_{f}} \rho_{v_{e}, v_{e}}\left(t_{n}\right)\left|\left\langle v_{e} \mid v_{f}\right\rangle\right|^{2} P_{K_{e}}(\cos \theta) j_{A}\left(v_{e}, v_{f}\right) .
$$

Используя выражения (23), (8) и (36), параметры Стокса $S_{1}$ и $S_{3}$ зондирующего излучения можно представить в виде:

$$
\begin{aligned}
& S_{1}=-\frac{C I_{p r}^{(0)}}{I_{p r}} \sum_{n}\left[\theta\left(t_{n}\right)-\theta\left(t_{n+1}\right)\right] e^{-\frac{t_{n}}{T}} r_{A}\left(t_{n}\right) \cos \varphi(t), \\
& S_{3}=-\frac{C I_{p r}^{(0)}}{I_{p r}} \sum_{n}\left[\theta\left(t_{n}\right)-\theta\left(t_{n+1}\right)\right] e^{-\frac{t_{n}}{T}} r_{D}\left(t_{n}\right) \cos \varphi(t),
\end{aligned}
$$

где

$$
r_{D}\left(t_{n}\right)=\frac{2}{5} R_{2}^{p r(D)}\left(t_{n}\right) D_{00}^{2}\left(t_{n}\right)
$$

И

$$
R_{K_{e}}^{p r(D)}\left(t_{n}\right)=\frac{1}{3} \sum_{v_{e}, v_{f}} \rho_{v_{e}, v_{e}}\left(t_{n}\right)\left|\left\langle v_{e} \mid v_{f}\right\rangle\right|^{2} P_{K_{e}}(\cos \theta) j_{D}\left(v_{e}, v_{f}\right) .
$$

Отметим, что суммирование по $n$ в выражениях (36), (39) и (40) записано в предположении, что к моменту каждого следующего импульса накачки сигнал от предыдущего импульса уже полностью затух. Это предположение соответствует условиям эксперимента [20,21]. 
Как видно из выражений (39) и (40), сигналы линейного дихроизма и двулучепреломления модулированы во времени по одинаковому закону $\cos \varphi(t)$, а полная интенсивность прошедшего зондирующего излучения $I_{p r}$ в (36) не модулирована. Поэтому при использовании модуляционной методики возможно не только разделение сигналов поглощения и дисперсии света, но и отделение поляризованного сигнала от сигнала полной интенсивности. Принимая во внимание, что период модуляции поляризации цуга лазерных импульсов в условиях эксперимента $[20,21]$ на три порядка превышал период следования лазерных импульсов, функции (39) и (40) можно считать периодическими и разложить их в ряд Фурье. При этом можно показать, что в сигналах (39) и (40) присутствуют только четные гармоники частоты модуляции $\Omega / 2$, причем коэффициетны разложения являются функциями Бесселя вида $2 J_{k}(\pi)$, где $k$ - порядок гармоники. Поэтому в работе [21] регистрировались сигналы, изменявшиеся по закону $\cos \Omega t$, т. е. находившиеся в квадратуре с опорным сигналом на двойной частоте модуляции $\Omega$.

\section{5. Выводы}

Таким образом, в настоящей работе были исследованы эффекты дихроизма и двулучепреломления в возбужденных состояниях многоатомных молекул при их исследовании методом накачка-зондирование. С помощью техники неприводимых тензорных операторов получены общие выражения для изменения интенсивности и состояния поляризации зондирующего импульса после прохождения раствора многоатомных молекул для любых исходных поляризаций импульса накачки и зондирующего импульса, как функция времени задержки между ними. Полученные выражения учитывают распад возбужденных состояний молекул за счет колебательной релаксации, вращательной диффузии и радиационных переходов. Полученные выражения содержат вклады от линейного дихроизма и двулучепреломления в возбужденных состояниях молекул, которые при определенных условиях могут наблюдаться одновременно. Показано, что вклады от линейного дихроизма и двулучепреломления в сигнал могут быть полностью разделены в эксперименте за счет надлежащего выбора анализатора поляризации зондирующего импульса, установленного перед фотодетектором. В частности, в геометрии, часто используемой для наблюдения оптического эффекта Керра, для наблюдения линейного дихроизма анализатор должен выделять сигнал, пропорциональный параметру Стокса $S_{1}$, а для наблюдения двулучепреломления анализатор должен выделять сигнал, пропорциональный параметру Стокса $S_{3}$. Полученные выражения применены для описания сигналов, получаемых при использовании поляризационно-модуляционной экспериментальной методики.

\section{Финансирование работы}

Авторы благодарят фонд Базис за финансовую поддержку в рамках гранта № 19-1-1-13-1.

\section{Конфликт интересов}

Авторы заявляют, что у них нет конфликта интересов.

\section{Список литературы}

[1] Mukamel S. Principles of Nonlinear Optical Spectroscopy. Oxford University Press, Oxford, New York, 2 edition, 1995. $543 \mathrm{p}$.

[2] Berera R., van Grondelle R., Kennis J.T.M. // Photosynth. Res. 2009. V. 101. P. 105. doi 10.1007/s11120-009-9454-y

[3] Boyd R.W. Nonlinear Optics. Elsevier, Academic Press, Rochester, Ottawa, 4 edition, 2020. 634 p.

[4] Cohen B., Hare P.M., Kohler B. // J. Am. Chem. Soc. 2003. V. 125. N 44. P. 13594. doi $10.1021 / \mathrm{ja} 035628 \mathrm{z}$

[5] Roberts G.M., Marroux H.J.B., Grubb M.P., Ashfold M.N.R., Orr-Ewing A.J. // J. Phys. Chem. A. 2014. V. 118. N 47. P. 11211. doi 10.1021/jp508501w

[6] Heiner Z., Roland T., Léonard J., Haacke S., Groma G.I. // J. Phys. Chem. B. 2017. V. 121. N 34. P. 8037. doi 10.1021/acs.jpcb.7b04753

[7] Lakowicz J.R. Topics in Fluorescence Spectroscopy. V. 5. NY: Plenum Press, 1997. 544 p.

[8] Vishwasrao H.D., Heikal A.A., Kasischke K.A., Webb W.W. // J. Biol. Chem. 2005. V. 208. N 26. P. 25119. doi 10.1074/jbc.M502475200

[9] Blacker T.S., Marsh R.J., Duchen M.R., Bain A.J. // Chem. Phys. 2013. V. 422. P. 184.

doi 10.1016/j.chemphys.2013.02.019

[10] Herbrich S., Al-Hadhuri T., Gericke K.-H., Shternin P.S., Smolin A.G., Vasyutinskii O.S. // J. Chem. Phys. 2015. V. 142. I. 2. doi $10.1063 / 1.4905140$

[11] Sasin M.E., Smolin A.G., Gericke K.-H., Tokunaga E., Vasyutinskii O.S. // Phys. Chem. Chem. Phys. 2018. V. 20. P. 19922. doi 10.1039/C8CP02708K

[12] Gorbunova I.A., Sasin M.E., Rubayo-Soneira J., Smolin A.G., Vasyutinskii O.S. // J. Phys. Chem. B. 2020. V. 124. N 47. P. 10682. doi 10.1021/acs.jpcb.0c07620

[13] Tan H.-S., Piletic I.R., Fayer M.D. // J. Opt. Soc. Am. B. 2005. V. 22. I. 9. P. 2009. doi 10.1364/JOSAB.22.002009

[14] Farrow D.A., Qian W., Smith E.R., Ferro A.A., Jonas D.M. // J. Chem. Phys. 2008. V. 128. P. 144510. doi 10.1063/1.2837471

[15] Fenn E.E., Wong D.B., Fayer M.D. // PNAS. 2009. V. 106. N 36. P. 15243. doi $10.1073 /$ pnas.0907875106

[16] Smith E.R., Jonas D.M. // J. Phys. Chem. A. 2011. V. 115. N 16. P. 4101. doi 10.1021/jp201928s

[17] Corrales M.E., Shternin P.S., Rubio-Lago L., R. de Nalda, Vasyutinskii O.S., Banares L. // J. Phys. Chem. Lett. 2016. V. 7. N 22. P. 4458. doi 10.1021/acs.jpclett.6b01874

[18] Rumble C., Vauthey E. // Phys. Chem. Chem. Phys. 2019. V. 21. P. 11797. doi 10.1039/C9CP00795D

[19] Hunger J., Roy S., Grechko M., Bonn M. // J. Phys. Chem. B. 2019. V. 123. N 8. P. 1831. doi 10.1021/acs.jpcb.8b10849

[20] Горбунова И.А., Сасин М.Э., Васютинский О.С. // Письма в ЖТФ. 2020. Т. 46. В. 4. С. 7; Gorbunova I.A., Sasin M.E., Vasyutinskii O.S. // Tech. Phys. Lett. 2020. V. 46. N 2. P. 158. doi 10.1134/S1063785020020212 
[21] Gorbunova I.A., Sasin M.E., Beltukov Ya.M., Semenov A.A., Vasyutinskii O.S. // Phys. Chem. Chem. Phys. 2020. V. 22. P. 18155. doi 10.1039/D0CP02496A

[22] Denicke S., Gericke K.-H., Smolin A.G., Shternin P.S., Vasyutinskii O.S. // J. Phys. Chem. A. 2010. V. 114. N 36. P. 9681. doi 10.1021/jp101403x

[23] Schaefer P.M., Kalinina S., Rueck A., von Arnim C.A.F., von Einem B. // Cytometry Part A. 2019. V. 95. N 1. P. 34. doi 10.1002/cyto.a.23597

[24] Waldeck D., Cross A.J., McDonald D.B., Fleming G.R. // J. Chem. Phys. 1981. V. 74. P. 3381. doi 10.1063/1.441491

[25] Zeug A., Rückmann I., Röder B. // J. Opt. B: Quantum Semiclass. Opt. 2001. V. 3. N 2. P. 251. doi 10.1088/1464$4266 / 3 / 2 / 377$

[26] Giraud G., Gordon C.M., Dunkin I.R., Wynne K. // J. Chem. Phys. 2003. V. 119. P. 464. doi 10.1063/1.1578056

[27] Lavorel B., Babilotte Ph., Karras G., Billard F., Hertz E., Faucher O. // Phys. Rev. A. 2016. V. 94. I. 4. P. 043422. doi 10.1103/PhysRevA.94.043422

[28] Picheyev B.V., Smolin A.G., Vasyutinskii O.S. // J. Phys. Chem. A. 1997. V. 101. N 41. P. 7614. doi 10.1021/jp971287y

[29] Cohen-Tannoudji C., Laloë F. // J. de Physique. 1967. V. 28. N 7. P. 505. doi 10.1051/jphys:01967002808-9072200/pdf

[30] Happer W. // Rev. Mod. Phys. 1972. V. 44. P. 169. doi 10.1103/RevModPhys.44.169

[31] Zare R.N. Angular Momentum. NY.: Wiley, 1988. 349 p.

[32] Ландау Л.Д., Либшии, Е.М. Квантовая механика: Нерелятивистская теория. Курс теоретической физики, Т. 3. М.: Наука, 4-е издание, 1989. 768 с.; Landau L.D., Lifshitz E.M. Quantum Mechanics: Non-relativistic Theory. Course of Theoretical Physics, V. 3. Pergamon, London, New York, 3 edition, 1977. $689 \mathrm{p}$.

[33] Варшалович Д.А., Москалев А.Н., Херсонский В.К. Квантовая теория углового момента. Л.: Наука, 1975. 439 с.; Varshalovich D.A., Moskalev A.N., Khersonskii V.K. Quantum Theory of Angular Momentum. NY. Singapore: World Scientifc, 1988. 528 p.

[34] Shternin P.S., Gericke K.-H., Vasyutinskii O.S. // Mol. Phys. 2010. V. 108. I. 7-9. P. 813. doi 10.1080/00268970903379221

[35] Александров Е.Б., Хвостенко Г.И., Чайка М.П. Интерференция атомных состояний. М.: Наука, 1991. 254 с.; Alexandrov E.B., Chaika M.P., Khvostenko G.I. Interference of Atomic States. NY: Springer-Verlag, 1993. 250 p.

[36] Herzberg G. Molecular Spectra Molecular Structure. I. Spectra of Diatomic Molecules. D. van Nostrand, 2 edition. 1950. $658 \mathrm{p}$. 